\title{
Airway abnormalities at flexible bronchoscopy in patients with chronic cough
}

\author{
S. Decalmer*, A. Woodcock*, M. Greaves" ${ }^{*}$ M. Howe and J. Smith*
}

ABSTRACT: The algorithms utilised in the diagnosis of chronic cough advocate sequential investigations and treatment trials for asthma-like syndromes, post-nasal drip and gastrooesophageal reflux disease; however, the role of bronchoscopy is unclear. In the present authors' specialist clinic (North West Lung Centre Cough Clinic, Manchester, UK), flexible bronchoscopy is included in the diagnostic work-up of patients with chronic unexplained cough. In a retrospective review, the authors report on their experiences.

Over an 18-month period, patients followed a diagnostic algorithm that included: chest radiography; pulmonary function; methacholine challenge; ear, nose and throat examination; and empirical reflux treatment. Where diagnosis remained elusive, bronchoscopy was performed.

A total of $\mathbf{8 2}$ bronchoscopies were carried out for the sole indication of chronic cough. Patient age (mean \pm SD) was $54.9 \pm 11.22$ yrs, with a median (range) cough duration of 5 (0.5-30) yrs. In nine $(11 \%)$ subjects, a diagnosis was made on inspection or biopsy. These included seven cases of tracheobronchopathia osteochondroplastica (TPO), one case of elongated uvula and one case of endobronchial amyloidosis. All TPO patients had early changes, with a typical nodular appearance to the tracheal cartilage, without significant airway obstruction. These subtle changes could not have been predicted from less invasive procedures and would have been missed without bronchoscopy.

Flexible bronchoscopy is indicated in persistent unexplained cough and may reveal contributing pathology.

\section{KEYWORDS: Bronchoscopy, cough, tracheobronchopathia osteochondroplastica}

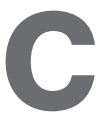

hronic cough, defined as a cough of $\geqslant 8$ weeks' duration, is a common and troublesome problem. In nonsmoking, immunocompetent individuals with normal lung function and chest radiograph, not currently taking angiotensin-converting enzyme (ACE) inhibitors, diagnostic protocols advocate sequential investigation of asthma-like syndromes, postnasal drip and gastro-oesophageal reflux disease [1-4]. Early studies, based on these anatomical diagnostic protocols $[1,5,6]$, suggested diagnostic successes approaching $100 \%$. In such studies, flexible bronchoscopy was rarely undertaken and, when carried out, found to add little diagnostically that could not have been predicted by other investigative modalities. Hence, its clinical usefulness in the assessment of patients with chronic persistent cough was questioned [6].

However, a group of patients is emerging in the setting of specialist cough clinics in which the cough evades diagnosis despite the appropriate use of diagnostic protocols and treatment trials. The term "idiopathic cough" has been coined to describe such individuals and this entity is recognised in $\leqslant 42 \%$ of cough clinic attendees $[1,5,7,8]$. Whether idiopathic cough exists or merely represents as yet undiagnosed or undertreated pathology is open to speculation, but such patients undoubtedly represent the more challenging end of the diagnostic spectrum.

In refractory cough patients, it has been suggested that the diagnostic yield from flexible bronchoscopy may be higher and may lead to diagnoses that might otherwise be missed should this procedure not be undertaken [9]. Hence, the American College of Chest Physicians and European Respiratory Society (ERS) guidelines list bronchoscopy as a further investigation for consideration in unexplained cough $[2,4]$.

The present authors review their experience with flexible bronchoscopy among patients with

\section{AFFILIATIONS}

*University of Manchester and North West Lung Centre, and, Depts of ${ }^{\#}$ Radiology, and "Histopathology, South Manchester University Hospitals Trust, Manchester, UK.

\section{CORRESPONDENCE}

J. Smith

North West Lung Research Centre

South Manchester University

Hospitals Trust

Southmoor Rd

Wythenshawe

Manchester M23 9LT UK

Fax: 441612915057

E-mail: jackyannsmith@hotmail.com

Received:

March 222007

Accepted after revision:

September 202007

SUPPORT STATEMENT

This study was supported by the North West Lung Centre Research Fund (Manchester, UK) and the Moulton Charitable Trust.

STATEMENT OF INTEREST None declared. 
chronic unexplained cough attending the North West Lung Centre Cough Clinic (Manchester, UK) over an 18-month period between January 2005 and June 2006. Patients are investigated as per diagnostic protocol guidelines [2, 4], including flexible bronchoscopy, once obvious causes of cough have been excluded.

\section{METHODS}

A retrospective review of records from attendees at the North West Lung Centre Cough Clinic was carried out. Patients who underwent flexible bronchoscopy between January 2005 and June 2006 were identified.

Patients were included if they had a cough of $\geqslant 8$ weeks of duration, which was persistent despite investigative protocols as outlined in the ERS guidelines for the diagnosis and management of chronic cough [2-4], and treatment trials for asthma, post-nasal drip and gastro-oesophageal reflux disease, where appropriate.

Patients were excluded if they were current smokers, if chest radiographic findings suggested a diagnosis, if other symptoms were an independent indication for bronchoscopy or if they were currently taking an ACE inhibitor.

\section{Statistics}

Logistic regression analysis was carried out with bronchoscopic findings as the dependant variable, and age, sex, smoking history and cough duration as predictor variables.

\section{RESULTS}

Over an 18-month period from January 1, 2005 to June 30, 2006, 125 new referrals were seen in the specialist cough clinic, $62 \%$ of which were tertiary referrals from other respiratory consultants. A total of 84 bronchoscopies were carried out. Two patients had independent indications for bronchoscopy (haemoptysis and oesophageal carcinoma with left lower lobe collapse on chest radiography) and were excluded from further analysis. In 82 patients, the only indication was chronic unexplained cough, the diagnosis of which had remained elusive despite investigative protocols and treatment trials. All patients tolerated the procedure and there were no complications.

The mean age of patients undergoing bronchoscopy was $54.9 \pm 11.22$ yrs, with median (range) cough duration of 5 (0.5-30) yrs. Approximately $67 \%(n=55)$ of patients were female and $40 \%$ were ex-smokers with median (range) smoking history of 12 (0.7-90) pack-yrs and abstinence of 20 (3-34) yrs, respectively.

\section{Bronchoscopic findings}

Appearances of the tracheobronchial tree were normal in 73 (89\%) patients. Seven patients had appearances consistent with a diagnosis of tracheobronchopathia osteochondroplastica (TPO), i.e. discreet nodules appearing on the cartilage rings, which were gritty in texture and difficult to biopsy. An unusually long uvula, touching the epiglottis, was seen in one patient (fig. 1).

One patient with known renal and cardiac AL amyloid was found to have endobronchial amyloidosis at biopsy, despite a negative thoracic computed tomography (CT) scan being performed to examine for pulmonary/endobronchial involvement in the presence of an unexplained cough (fig. 2).

Three patients with normal bronchoscopic appearances of the airways were noted to have vocal cord nodules. Bronchoscopic abnormalities were not significantly predicted by $\operatorname{sex}(\mathrm{p}=0.77)$, age $(p=0.87)$, duration of cough $(p=0.12)$ or smoking history $(\mathrm{p}=0.43)$.

All patients with bronchoscopic abnormalities had undergone a series of investigations and treatment trials. Table 1 summarises the investigation findings for each subject. Where methacholine challenge was positive, inhaled corticosteroids were administered; evidence of post-nasal drip was treated with antihistamines and nasal steroids; acid reflux was treated with twice daily proton pump inhibitors and nocturnal $\mathrm{H}_{2}$ blockers; and nonacid reflux was treated with a prokinetic agent. Treatment trials were for $\geqslant 8$ weeks. Where more than one potential cause was identified, treatment trials were concomitant. None of these subjects responded to treatment trials.

\section{Subjects with TPO}

The characteristics of the seven subjects with TPO were similar to the bronchoscopy cohort as a whole. The mean age was $53.7 \pm 9.0 \mathrm{yrs}$, five $(71 \%)$ were female, three $(43 \%)$ were exsmokers and median (range) cough duration was 5.0 (1.5-20) yrs.

All CT changes of TPO were extremely subtle and easily overlooked.

\section{DISCUSSION}

Flexible bronchoscopy is a widely available technique for direct visualisation and sampling of the airways, and is associated with low morbidity and mortality. The current study is the largest to date to report on findings in chronic unexplained cough. In the present group of patients attending the authors' tertiary referral clinic, flexible bronchoscopy resulted in identification of important abnormalities in $11 \%$ of the cases (TPO in 8.5\%, endobronchial amyloidosis in 1.2\% and long uvula in 1.2\%), which would have been missed had visualisation of the airways been omitted.

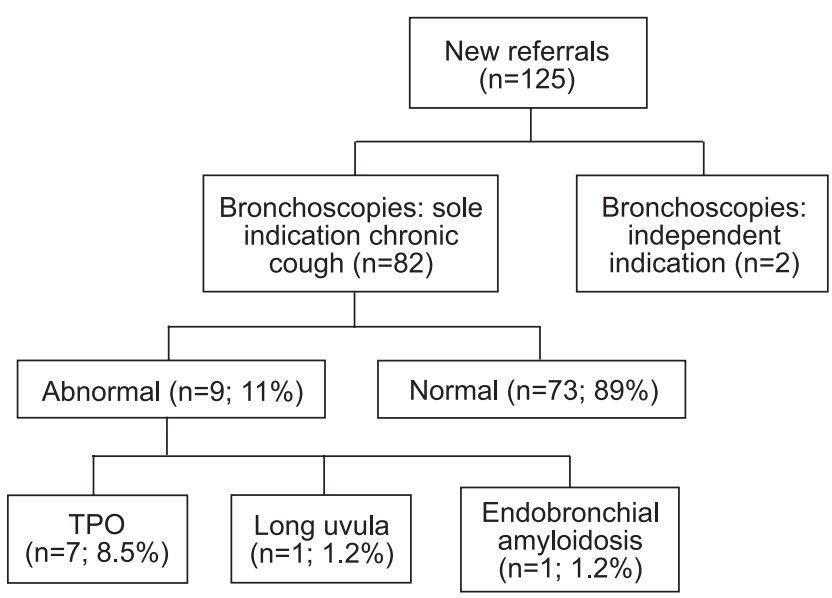

FIGURE 1. Summary of bronchoscopic abnormalities found in patients with chronic unexplained cough. TPO: tracheobronchopathia osteochondroplastica. 

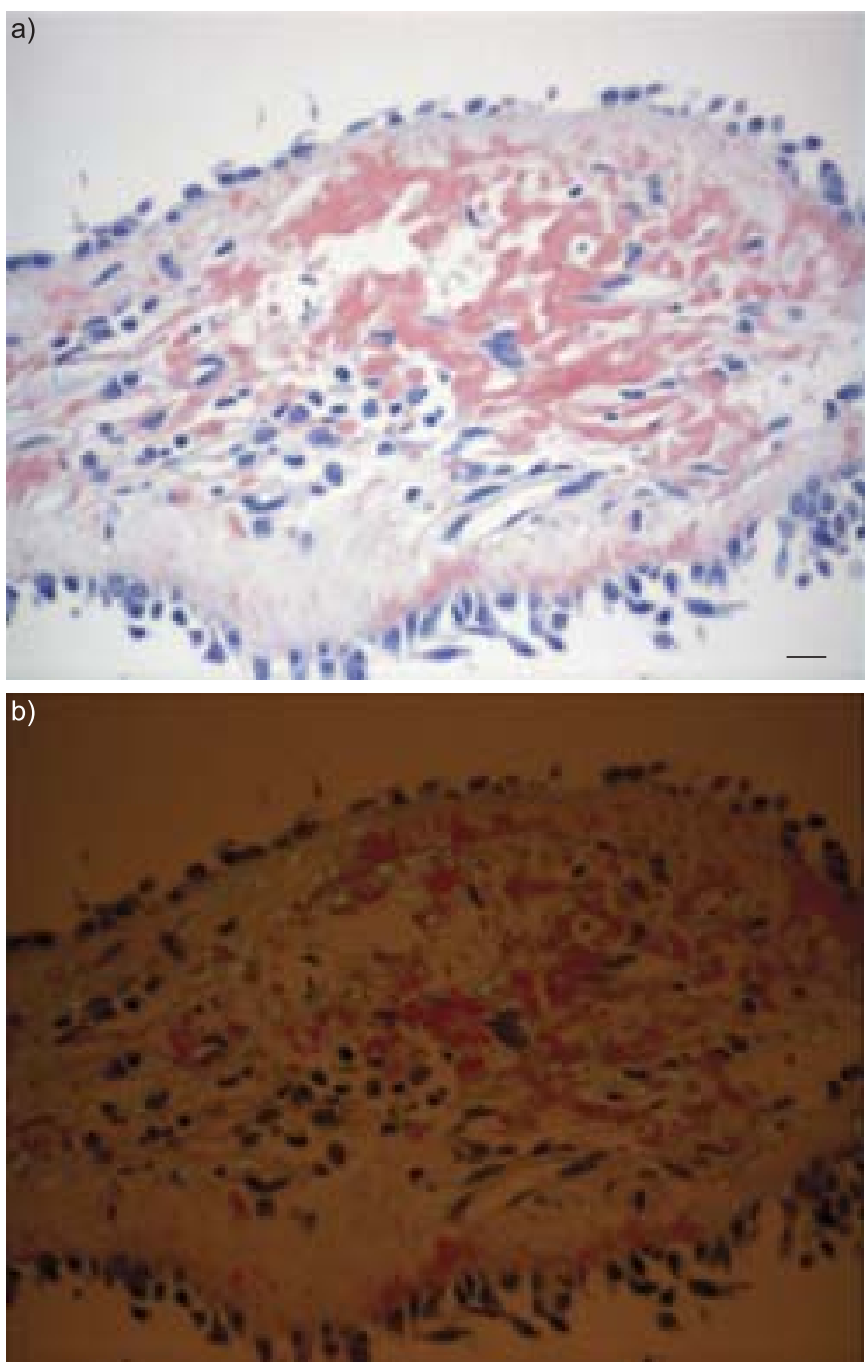

FIGURE 2. Microscopic appearance of endobronchial amyloid: a) stained with Congo red; and b) the amyloid deposits show apple green birefringence under polarised light. Scale bar $=50 \mu \mathrm{m}$.

Pioneering work by IRWIN et al. [1] in the 1980s led to the widespread implementation of a diagnostic algorithm, which is valuable in guiding diagnosis and management in unselected patients with chronic cough. Among such patients, bronchoscopy provided a low diagnostic yield, adding little information, all predictable by less invasive means $[1,6]$. However, the role of bronchoscopy is less clear in patients referred to tertiary clinics with unexplained cough. In the present authors' specialist clinic, bronchoscopy has been incorporated into the diagnostic algorithm and has shown a significant yield in this group of patients.

It is important to emphasise that almost two thirds of these patients were referred by other respiratory consultants following a series of investigations and failed treatment trials. While the present authors' observations cannot be applied to the chronic cough population as a whole, bronchoscopy appears to have a significant role in diagnosis in this select group of patients.

TPO was the most common bronchoscopic abnormality. All the patients showed early TPO with no airflow obstruction on pulmonary function testing and no radiographic changes. In both the present study and that of SEN and WALSH [9], TPO appeared over-represented (8-8.5\%) among chronic cough patients; the existing literature suggests it had been observed in $0.4 \%$ of bronchoscopies $[9,10]$. Other bronchoscopic studies in chronic cough patients make no mention of this phenomenon [11-15]; however, these were predominantly focused on lavage findings and literature suggests that such abnormalities may be overlooked or their significance misinterpreted in up to one third of patients [10].

TPO is a rare benign disorder of unknown aetiology and characterised by cartilaginous nodules projecting into the airway lumen from the anterior and lateral walls of the tracheobronchial tree (fig. 3) [10, 16]. The posterior membranous wall is spared and, if involvement is seen, an alternative diagnosis is likely. If nodules affect the posterior wall and are friable in nature, a diagnosis of endobronchial amyloidosis must be excluded $[16,17]$.

The true incidence of TPO is unknown due to nonspecific symptoms, and identification may occur coincidentally at autopsy, on magnetic resonance imaging or at intubation for unrelated reasons $[10,16]$. There appears to be a male predominance and the condition usually presents in the sixth or seventh decade of life $[10,16]$.

Diagnosis is based on typical bronchoscopic appearances and biopsy of the lesions is difficult due to their bony nature. When appearances are typical, biopsy is generally thought not to be essential for diagnosis [10,16], although this is refuted by one group who feel histological confirmation is necessary [18]. Histology, when available, reveals cartilaginous elements within the submucosa $[10,16]$. Chest radiographs are often normal or display only subtle scalloping of the inner tracheal mucosa [10, 19]. CT may demonstrate tracheal narrowing and nodularity, with or without calcification, but, again, may be normal $[10,19,20]$. Flow-volume loops are often normal in early or mild disease but, with progression, reveal variable degrees of intra- and extra-thoracic central airway obstruction [20, 21].

TPO tends to be very slowly progressive, but indolent nodule proliferation can result in airway narrowing and symptoms of obstruction [20]. Tracheal trauma may increase the rate of progression and, only rarely, a rapidly progressive form can result in severe tracheal stenosis and death [21].

A total of $88 \%$ of patients with TPO complain of one or more respiratory symptom, including sputum production, dyspnoea, wheeze, stridor and hoarseness. Cough is the most common, reported in $54 \%$. It is hypothesised that this is due to turbulent air flow, increased cough reflex sensitivity and impaired ciliary clearance of sputum [10, 22].

Respiratory tract infections are common and haemoptysis can occur if the mucosa overlying the nodules ulcerates [10, 16, 21].

No specific treatment exists for TPO but laser ablation, debulking, dilation or surgical splinting of the airway has been used for symptomatic relief of obstructive symptoms [23]. Hydration, humidification and mucolytics may also be helpful, along with prompt antibiotic use for microbial infection [10, 22]. As no specific treatments exist, TPO cannot be confirmed 
TABLE 1 Summary of investigations performed in subjects with abnormal bronchoscopic findings

\begin{tabular}{|c|c|c|c|c|c|c|c|}
\hline Subject & Diagnosis & FEV 1 & FVC & $\begin{array}{l}\text { Methacholine } \\
\text { challenge }\end{array}$ & Reflux episodes ${ }^{\#}$ & Demeester score & ENT examination \\
\hline 1 & TPO & 117 & 112 & Negative & 72 & 0.8 & Normal \\
\hline 2 & TPO & 94 & 108 & Mildly positive & Not tolerated ${ }^{+}$ & Not tolerated ${ }^{+}$ & Normal \\
\hline 5 & TPO & 107 & 107 & Negative & 78 & 1.4 & Post-nasal drip \\
\hline 6 & TPO & 93 & 112 & Mildly positive & 62 & 9.4 & Normal \\
\hline 7 & TPO & 110 & 111 & Negative & 56 & 3.9 & Normal \\
\hline 8 & Long uvula & 135 & 136 & Negative & 2 & 0.4 & Normal \\
\hline 9 & Amyloidosis & 95 & 98 & Negative & Not tolerated ${ }^{\S}$ & Not tolerated ${ }^{\S}$ & Post-nasal drip \\
\hline
\end{tabular}

\% pred: \% predicted; ENT: ear, nose and throat; FEV1: forced expiratory volume in one second; FVC: forced vital capacity; TPO: tracheobronchopathia osteochondroplastica. *: normal can be defined as $<73$ episodes; " : normal can be defined as a score $<14.7 ;^{+}$: mild gastritis at gastroscopy and, therefore, treated for acid reflux disease; ": gastroscopy showed minimal peristalsis and biopsy confirmed amyloid infiltration.

as the cause of cough in the conventional manner, i.e. by trials of treatment. Therefore, it is possible that TPO is merely a comorbidity but the over-representation of this condition in
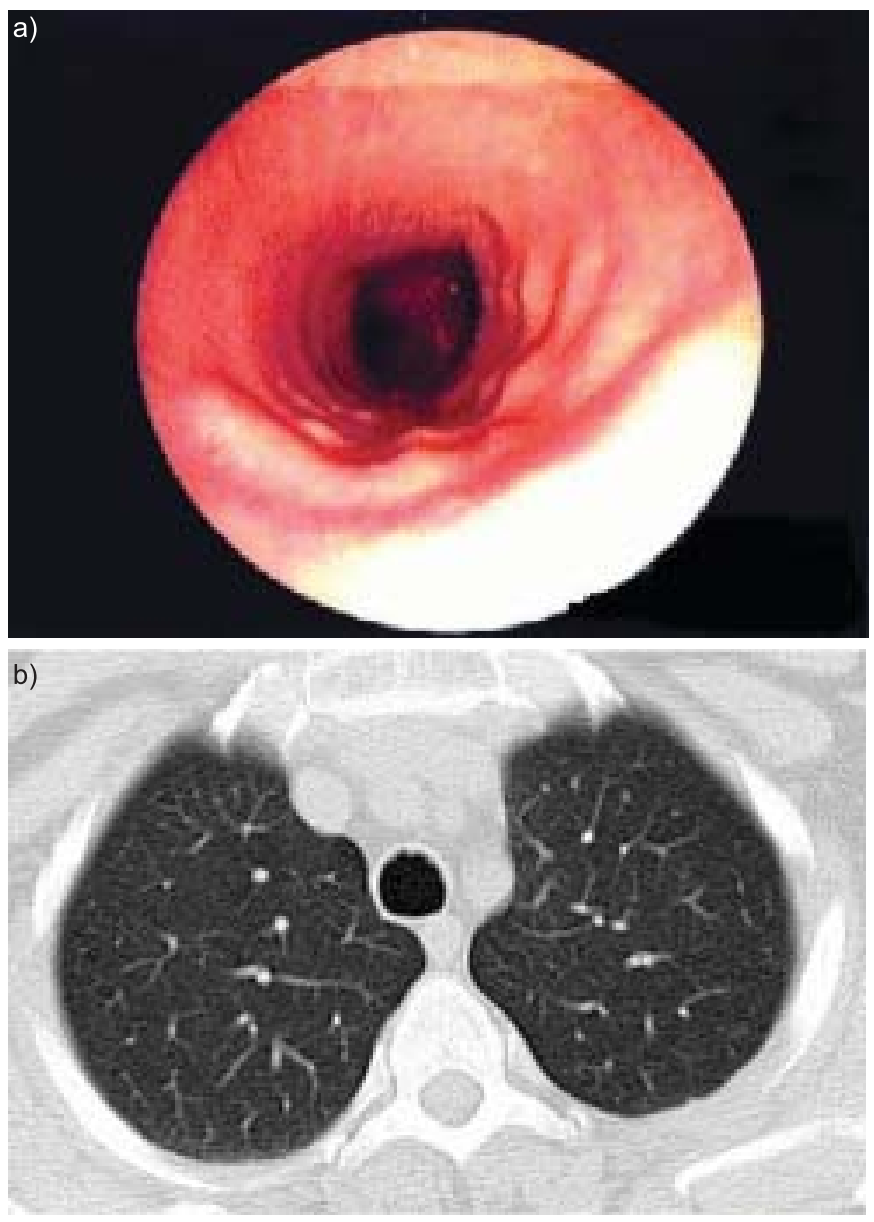

FIGURE 3. a) Bronchoscopic and b) computed tomography (CT) appearances of tracheobronchopathia osteochondroplastica. Note the discrete nodules lying along the cartilage rings, barely visible on the CT scan. patients where other causes of cough have been excluded suggests that this is not the case.

It might be argued that the bronchoscopic diagnosis of an essentially untreatable condition is of little clinical value. Patients with chronic cough have often been referred to many doctors in several specialties in the hope of finding the cause of their symptom and have high levels of associated anxiety and depression [24, 25]. Identifying a possible explanation may reduce subsequent referrals and investigations and may even help to alleviate some of the associated concerns. Once other causes for cough have been excluded with the diagnostic and treatment protocols, attempts can be made to provide symptomatic relief in such individuals through antitussive trials.

Vocal cord nodules were observed in three patients. These are thought to result from mechanical stress due to voice overuse, misuse or abuse [26]. It is conceivable that these nodules are a consequence of repeated trauma induced by cough.

In addition to the findings of the present study, many other abnormalities of the tracheobronchial tree, which can be responsible for chronic cough, may be overlooked without direct visualisation of the airways. Benign and malignant tracheal tumours often present with cough and may go unrecognised on chest radiography [27]. Tracheomalacia, resulting in a distinctive "seal bark" cough can be diagnosed when expiratory manoeuvres result in airway collapse and a scabbard shape adopted by the malacic segment. This can be congenital, due to cartilage abnormalities or idiopathic giant trachea (Mounier-Kuhn Syndrome) or result from trauma, compression (cysts, vascular rings, aortic aneurysms, thymomas and malignancy) or chronic inflammation (relapsing polychondritis) $[28,29]$.

Tracheobronchial abnormalities may also be features in tuberculosis, sarcoidosis, Wegner's granulomatosis and papillomatosis and marked mucopurulent secretions seen at bronchoscopy might lead to a diagnosis of suppurative lung disease even with a minimal history of sputum production. 
Bronchoscopy also gives the opportunity for evaluation of the vocal cords, identifying patients with vocal cord dysfunction who may benefit from speech therapy intervention.

In summary, it has been demonstrated that bronchoscopy is a useful technique in a select group of patients with unexplained chronic persistent cough despite investigation and treatment trials. Abnormalities (mainly tracheobronchopathia osteochondroplastica) were identified in $11 \%$ of patients who would have been missed had the procedure not been undertaken. Therefore, in patients with chronic unexplained cough, bronchoscopy should be performed.

\section{ACKNOWLEDGEMENTS}

The authors would like to thank the Bronchoscopy Dept at the North West Lung Centre (Manchester, UK), especially J. Martin (nurse bronchoscopist) and J. Bowker (bronchoscopy nurse).

\section{REFERENCES}

1 Irwin RS, Corrao WM, Pratter MR. Chronic persistent cough in the adult: the spectrum and frequency of causes and successful outcome of specific therapy. Am Rev Respir Dis 1981; 123: 413-417.

2 Morice AH, Fontana GA, Sovijarvi AR, et al. The diagnosis and management of chronic cough. Eur Respir J 2004; 24: 481-492.

3 Morice AH, McGarvey L, Pavord I, British Thoracic Society Cough Guideline Group. Recommendations for the management of cough in adults. Thorax 2006; 61: Suppl. 1, i1-i24.

4 Irwin RS, Baumann MH, Bolser DC, et al. Diagnosis and management of cough executive summary: ACCP evidence-based clinical practice guidelines. Chest 2006; 129: Suppl. 1, S1-S23.

5 Poe RH, Harder RV, Israel RH, Kallay MC. Chronic persistent cough. Experience in diagnosis and outcome using an anatomic diagnostic protocol. Chest 1989; 95: 723-728.

6 Barnes TW, Afessa B, Swanson KL, Lim KG. The clinical utility of flexible bronchoscopy in the evaluation of chronic cough. Chest 2004; 126: 268-272.

7 McGarvey LP, Heaney LG, MacMahon J. A retrospective survey of diagnosis and management of patients presenting with chronic cough to a general chest clinic. Int J Clin Pract 1998; 52: 158-161.

8 Haque RA, Usmani OS, Barnes PJ. Chronic idiopathic cough: a discrete clinical entity? Chest 2005; 127: 1710-1713.

9 Sen RP, Walsh TE. Fiberoptic bronchoscopy for refractory cough. Chest 1991; 99: 33-35.

10 Lundgren R, Stjernberg NL. Tracheobronchopathia osteochondroplastica. A clinical bronchoscopic and spirometric study. Chest 1981; 80: 706-709.

11 McGarvey LP, Forsythe P, Heaney LG, MacMahon J, Ennis M. Bronchoalveolar lavage findings in patients with chronic nonproductive cough. Eur Respir J 1999; 13: 59-65.
12 Niimi A, Chung KF. Airway inflammation and remodelling changes in patients with chronic cough: do they tell us about the cause of cough? Pulm Pharmacol Ther 2004; 17: 441-446.

13 Niimi A, Torrego A, Nicholson AG, Cosio BG, Oates TB, Chung KF. Nature of airway inflammation and remodeling in chronic cough. J Allergy Clin Immunol 2005; 116: 565-570.

14 Lee SY, Cho JY, Shim JJ, et al. Airway inflammation as an assessment of chronic nonproductive cough. Chest 2001; 120: 1114-1120.

15 Birring SS, Morgan AJ, Prudon B, et al. Respiratory symptoms in patients with treated hypothyroidism and inflammatory bowel disease. Thorax 2003; 58: 533-536.

16 Leske V, Lazor R, Coetmeur D, et al. Tracheobronchopathia osteochondroplastica: a study of 41 patients. Medicine (Baltimore) 2001; 80: 378-390.

17 Thomas D, Stonell C, Hasan K. Tracheobronchopathia osteoplastica: incidental finding at tracheal intubation. $\mathrm{Br} J$ Anaesth 2001; 87: 515-517.

18 Pérez-Rodríguez E, Núñez N, Alvarado C, et al. Diagnosis of tracheopathia osteochondroplastica. Chest 1990; 97: 763.

19 Restrepo S, Pandit M, Villamil MA, Rojas IC, Perez JM, Gascue A. Tracheobronchopathia osteochondroplastica: helical CT findings in 4 cases. J Thorac Imaging 2004; 19: 112-116.

20 Meyer CN, Døssing M, Broholm H. Tracheobronchopathia osteochondroplastica. Respir Med 1997; 91: 499-502.

21 Molloy AR, McMahon JN. Rapid progression of tracheal stenosis associated with tracheopathia osteochondroplastica. Intensive Care Med 1988; 15: 60-62.

22 Chen AY, Donovan DT. Impaired ciliary clearance from tracheopathia osteoplastica of the upper respiratory tract. Otolaryngol Head Neck Surg 1997; 117: S102-S104.

23 Tibesar RJ, Edell ES. Tracheopathia osteoplastica: effective long-term management. Otolaryngol Head Neck Surg 2003; 129: 303-304.

24 Dicpinigaitis PV, Tso R, Banauch G. Prevalence of depressive symptoms among patients with chronic cough. Chest 2006; 130: 1839-1843.

25 McGarvey LP, Carton C, Gamble LA, et al. Prevalence of psychomorbidity among patients with chronic cough. Cough 2006; 2: 4.

26 Johns MM. Update on the etiology, diagnosis, and treatment of vocal fold nodules, polyps, and cysts. Curr Opin Otolaryngol Head Neck Surg 2003; 11: 456-461.

27 Ko JM, Jung JI, Park SH, et al. Benign tumors of the tracheobronchial tree: CT-pathologic correlation. AJR Am J Roentgenol 2006; 186: 1304-1313.

28 Carden KA, Boiselle PM, Waltz DA, Ernst A. Tracheomalacia and tracheobronchomalacia in children and adults: an in-depth review. Chest 2005; 127: 984-1005.

29 Maimon N, Marras T, Hwang D, Paul N, Keshavjee S, Chan CK. A 46-year-old female with dyspnoea, stridor and chronic cough. Eur Respir J 2006; 28: 666-669. 